\title{
PREPARATION OF SILICONE-MODIFIED ACRYLATE LATEX AND ITS APPLICATION FOR LOW-EMISSION PRINTING OF PET FIBRE
}

\author{
Li Ai ${ }^{1}$, Hongmei Cao ${ }^{1,2}$, Yawei Zhu ${ }^{1,3, *}$ \\ ${ }^{1}$ College of Textile and Clothing Engineering, Soochow University, Suzhou, Jiangsu 215021, China \\ ${ }^{2}$ Changzhou Vocational Institute of Textile and Garment, Changzhou, Jiangsu 213164, China \\ ${ }^{3}$ National Engineering Laboratory for the Modern Silk (Suzhou), Suzhou, Jiangsu 215123, China \\ ${ }^{*}$ Corresponding author. Email yaweizhu@suda.edu.cn. Tel.: +86 13812636806
}

\begin{abstract}
:
Attempts were made to find a more environmentally friendly technique for the printing of polyester (PET) fabric, acting as an alternative to a usual disperse dye direct-printing process by using a plenty of water and salt and producing effluent contaminants. The low-emission printing technique includes the recipe containing disperse dye paste, synthetic thickener and the water-based silicone-modified acrylate and high-temperature curing process. The water-based silicone-modified acrylate for adhesive coating of polyester (PET) fibres was synthesized using butyl acrylate (BA), eight four methyl siloxane (D4), acrylonitrile (AN), styrene (St), methyl acrylic acid (MAA) and $N$-methylol acrylamide (NMA). The results showed that the silicone-modified acrylate adhesive could increase the percentages of dye fixation and the colour strength. The superior colour fastness ( $\geq l e v e l 4)$ with the low-emission printing process was realized. The wastewater stream produced by the technique had a residual dye concentration of $2.62 \mathrm{mg} / \mathrm{L}$, which was reduced by approximately 19 times that produced by traditional direct printing. The effluent wastewater drainage was reduced by $76.9 \%$.
\end{abstract}

\section{Keywords:}

silicone-modified acrylate, colour fastness, low emission, direct printing

\section{Introduction}

Direct printing is the most extensively used technique for textiles due to its many advantages over other dye printing methods that include being rich in colour scheme, having soft handle and good colour fastness. Nevertheless, it also exhibits more water consumption and wastewater pollution.

Pigment printing is broadly applied in all types of textile fibres because pigment adheres on the fibres, without reaction with or deposit between fibres. It also has advantages of being a simple process with low water consumption and residue emissions. However, the disadvantages of pigment printing include less bright colour, harder handle and unsatisfactory colour fastness [1].

Pigment printing pastes contain a thickening agent and an adhesive. The colour fastness depends on the adhesive, which is not beneficial to handle. The synthesis of different adhesive types has been the research on pigment printing, primarily with polybutadiene, polyacrylate and polyurethane adhesive [2, 3]. When polybutadiene binder is applied to fibre, the merits are outstanding, i.e., soft handle, excellent elasticity and low cost [4, 5]. However, the obvious disadvantage of this adhesive is the susceptibility to yellowing because of oxidation, and the viscosity and light fastness are worse than with polyacrylate. The core-shell adhesive of polyacrylate modified by silicone and polyurethane is also widely used because of its favourable properties; the former has a soft handle, improved colour fastness and better wet and dry rubbing fastness[3, 6-8], while the latter has a high elasticity, heat resistance, good water-vapour permeability, a soft handle and low cost $[9,10]$. The addition of crosslinking to print pastes improves the combinative fastness between the coating and the fibre. By forming a three-dimensional network structure between the crosslinking and fibre molecules, performance properties such as the soaping and rubbing fastness are improved. However, the fibre yellows easily and has a hard handle when curing at high temperatures [11].

Herein, by combining the advantages of direct and pigment printing, we propose the new way of low-emission printing, which is an adjustment to the printed medium consisting of liquid disperse dyes, the thickener and core-shell adhesive of polyacrylate modified by silicone. The primary purpose of low-emission printing is to reduce the burden of print post processing and obtain good colour fastness and a soft handle in the fabric. Low-emission printing aims to solve the problems of high wastewater discharge and waste residue production of disperse dye printing, an outcome that possesses a high potential economic value and ecological significance.

\section{Materials and methods}

\subsection{Materials}

In the present study, polyester fibre was supplied by Jiangsu Cheng-Hong Group (China). Synthetic thickener PTF-A was 
purchased from Foshan Yimei Chemical Co., Ltd. (China). Sodium alginate was supplied by Qingdao Bright Moon Group (Shandong Province, China). Sodium carboxymethyl cellulose (CMC) was supplied by Group Chemical Reagent Co., Ltd. (Shanghai China). Sodium hyposulfite was supplied by Wuxi Sheng Chemical Co., Ltd. (China). Synthetic detergent was supplied by Shanghai White Cat Specialty Chemicals Co., Ltd. (China). Diethylene glycol monobutyl ether was supplied by Group Chemical Fertilizer Co., Ltd. Eight four methyl siloxane (D4), butyl acrylate (BA), acrylonitrile (AN), styrene (St), methyl acrylic acid (MAA), N-methylol acrylamide (NMA), and $\mathrm{N}, \mathrm{N}$-dimethylformamide (DMF) were of analytical grade, purchased from Shanghai Lingfeng Chemical Reagent Co., Ltd. (Shanghai, China). Fatty alcohol polyoxyethylene ether sodium sulfate (AES), heterogeneous 13 alcohol ether (To7 ), Dispersant $2000 \mathrm{~A}$ and polyacrylic acid sodium were of industrial grade.

\subsection{Preparation of liquid disperse dye}

C.I. disperse blue 79 was supplied by Jiangsu Yabang Dyestuff Co., Ltd. (China). The dye structure is shown in Fig. 1. C.I. disperse blue $79(40 \mathrm{~g})$, disperse agent $(4 \mathrm{~g})$, a non-ionic surfactant $(1 \mathrm{~g})$ and water $(155 \mathrm{ml})$ was fully mixed and ground for $3 \mathrm{~h}$ in the homemade zirconia grinding machine. The average size of the dye particles was $1 \mu \mathrm{m}$ after filtering.<smiles>CCOc1cc(N=Nc2c(Br)cc([N+](=O)[O-])cc2[N+](=O)[O-])c(NC(C)=O)cc1N(CCOC(C)=O)CCOC(C)=O</smiles>

Figure 1. C.I. disperse blue 79

\subsection{Preparation of silicone-modified acrylate emulsion}

With butyl acrylate, St, AN and D4, methacrylic acid and NMA as raw materials, five types of silicone-modified acrylate emulsion were prepared by semi-continuous emulsion polymerization according to the quality of the raw material (Table 1). In the process of synthesis, the mixture of AES and To- 7 was used as an emulsifier and $\left(\mathrm{NH}_{4}\right)_{2} \mathrm{~S}_{2} \mathrm{O}_{8}$ as an initiator. Out of $33 \mathrm{ml}$ distilled water, $0.33 \mathrm{~g}\left(\mathrm{NH}_{4}\right)_{2} \mathrm{~S}_{2} \mathrm{O}_{8}$ was added to a clear $250 \mathrm{ml}$ four-neck round-bottom flask with a stirrer. One-third of the premixed monomers (see Table 1 for the composition of monomers) was put into four-neck round-bottom flask, and with adding D4 into the rest of premixed monomers, it was mixed and put into a 100$\mathrm{ml}$ dropping funnel. When the liquid in the flask became blue, the mixed monomers and initiator were added to the flask over $0.5 \mathrm{~h}$ to $1 \mathrm{~h}$. After the drop feeling was ended, the thermal insulation reaction was completed for $2 h$ at the design temperature. The product then was cooled to room temperature. The emulsion membrane was obtained at $50^{\circ} \mathrm{C}$. The chemical formula for the binder and a schematic are presented in Fig. 2.

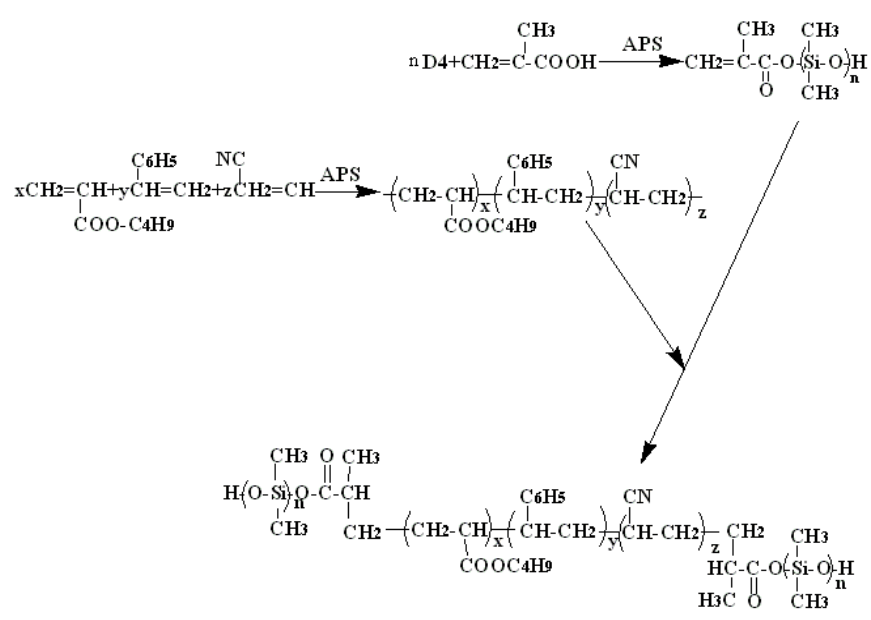

Figure 2. Chemical formula and a schematic presentation of siliconemodified acrylate emulsion

\subsection{Printing}

The low-emission printing paste containing liquid disperse dye ( $2 \%$ disperse dye blue 79 ), synthetic thickener (1\% PTF-A synthetic thickener), silicone-modified acrylate adhesive (2\%) and sufficient water was well stirred. The process is as follows:

$$
\begin{aligned}
& \text { Printing } \rightarrow \text { Drying } \quad\left(70^{\circ} \mathrm{C}\right) \rightarrow \text { Baking } \quad\left(180^{\circ} \mathrm{C}, \quad 60 \mathrm{~s}\right) \rightarrow \text { Water } \\
& \text { washing }\left(50^{\circ} \mathrm{C}\right) \rightarrow \text { Drying }
\end{aligned}
$$

The direct-printing paste containing commercial disperse dye ( $2 \%$ C.I. disperse blue 79 ), thickener ( $3 \%$ CMC (directprinting-1) or $4 \%$ sodium alginate (direct-printing-2)) and sufficient water was well stirred. The process is as follows:

Printing $\rightarrow$ Drying $\left(70^{\circ} \mathrm{C}\right) \rightarrow$ Baking $\left(180^{\circ} \mathrm{C}, 60 \mathrm{~s}\right) \rightarrow$ Reduction clearing $\left(85^{\circ} \mathrm{C}, 15 \mathrm{~min}\right) \rightarrow$ Water washing $\rightarrow$ Drying

\begin{tabular}{|c|c|c|c|c|}
\hline \multirow{2}{*}{$\begin{array}{c}\text { Functional } \\
\text { polymers }\end{array}$} & \multirow{2}{*}{$m(B A): m(s t y r e n e): m(A N): m(D 4): m(M A): m(N-M A N)$} & \multicolumn{2}{|c|}{ Membrane properties } & \multirow{2}{*}{$\begin{array}{c}\text { Elasticity } \\
\text { and } \\
\text { flexibility }\end{array}$} \\
\hline & & $\begin{array}{c}\text { Breaking } \\
\text { strength }(\mathrm{cN})\end{array}$ & $\begin{array}{c}\text { Breaking } \\
\text { elongation (\%) }\end{array}$ & \\
\hline A & $17.42: 0: 0: 15.58: 0: 0$ & - & - & Adhesive \\
\hline $\mathrm{B}$ & 20.00:2.00:0:10.00:0.50:0.50 & 82.97 & 1007.59 & Good \\
\hline $\mathrm{C}$ & $20.50: 3.50: 2.00: 6.00: 0.50: 0.50$ & 176.23 & 1103.76 & Better \\
\hline $\mathrm{D}$ & $11.00: 6.50: 6.00: 7.50: 0.80: 1.20$ & 1258.18 & 144.58 & Hard \\
\hline$E$ & $9.15: 7.65: 6.75: 5.95: 1.70: 1.80$ & 5646.80 & 5.60 & Harder \\
\hline
\end{tabular}

Table 1. Relationship between the monomer composition and properties of silicone-modified acrylate emulsion membrane. 


\subsection{Measurements of K/S and percentage of dye fixation}

The samples were analysed by measuring the reflectance curve between 400 and $700 \mathrm{~nm}$ using a Datacolour instrument (UltraScan XE; Hunter Lab Co., USA) equipped with a pulsed xenon lamp filtered to approximate D65 diffuse illumination and ten viewing geometries to evaluate the colour yield. The RF value of the printed sample was used to determine the ratio of the $K / S$ value before and after washing or soaping via Formula (1).

$$
\mathrm{RF}=[\mathrm{K} / \mathrm{S} 2] /[\mathrm{K} / \mathrm{S} 1]
$$

(K/S) 1-the value of $K / S$ before soaping.

$(K / S) 2$ - the value of $K / S$ after soaping.

First, a working curve reflecting the relationship between the value of absorbance of dye solution and its dye concentration was plotted, and then using this working curve and through measurement of the value of absorbance of extract at the maximum wavelength with a UV-Vis spectrophotometer (TU1810; Beijing's Analysis of General Instrument Co., Ltd.), the dye concentration in fabric could be obtained. Second, the equivalent area of two samples (printing fibres and no-printing fibres) were closed together by stitching, the disperse dyes were fixed with hot air using the M. Tenter continuous setting and curing machine (Rabbit Co., Taiwan) and dye fixation samples (printing fibres) and dye sublimation samples (noprinting fibres) were prepped separately. Third, the dye fixation samples were completed with a soaping process that removed residual materials. Fourth, the dye in the fibre was extracted with DMF and water bath at $80^{\circ} \mathrm{C}$. Fifth, three samples bath were gathered; the concentration of dye in the fibre ( $C f$, printing fibres) could be obtained. Consequently, the concentration of dye in the fibre ( $C s$, no-printing fibres) and dye in the soap residual bath $(\mathrm{Cr})$ could be measured and calculated by a working curve. The percentage of dye fixation $(F \%)$ and the percentage of dye sublimation $(S \%)$ were calculated using Equ. (2) and Equ. (3).

$$
\begin{aligned}
& F \%=[C f /(C f+C r+C s)] \times 100 \\
& S \%=[C s /(C f+C r+C s)] \times 100
\end{aligned}
$$

\subsection{Measurement of washing and rubbing fastness}

The washing fastness test was performed according to the standard (ISO 105-C02:2013) with WashTec-P washing fastness tester (ROM Chase International Company, USA).

The rubbing fastness test was performed according to the standard (ISO 105-X12:2016) using a Model 670 type friction fastness machine (James $\mathrm{H}$. Heal and Company, USA).

\subsection{Surface characterization}

FTIR spectra of the silicone-modified acrylate membrane were used to characterize its chemical structure. The spectrometer is designed to record the IR spectra of samples in the frequency range of $650-4000 \mathrm{~cm}^{-1}$.
SEM (S-4800; Hitachi, Japan) was used to intuitively observe the surface morphology of printed polyester fabric, which could be used to evaluate the influence of the functional polymer. SEM images shows that the film of functional polymer was formed on the fabric.

\section{Results and discussion}

\subsection{FTIR of silicone-modified acrylate emulsion membrane}

FTIR spectra of silicone-modified acrylate emulsion membrane are shown in Fig. 3, where 2961 and $2872 \mathrm{~cm}^{-1}$ are the $\mathrm{C}-\mathrm{H}$ stretching vibrating absorbing peaks of $-\mathrm{CH}_{3}$ and $-\mathrm{CH}_{2}$ in the polymer chain. $1732 \mathrm{~cm}^{-1}$ is the $-\mathrm{C}=\mathrm{O}$ stretching vibrating peak. $1057 \mathrm{~cm}^{-1}$ is Si-O-Si absorbing peaks. FTIR of samples A, B and $C$ illustrated that D4 had done the polymerization. $2241 \mathrm{~cm}^{-1}$ is the $-\mathrm{C} \equiv \mathrm{N}$ stretching vibrating absorbing peak, which illustrated that AN had done the polymerization, as shown in FTIR spectra of sample D. FTIR spectra of sample E show that D4 had little done the polymerization.

\subsection{Effect of silicone-modified acrylate emulsion adhesive in printing}

When the baking temperature was $190^{\circ} \mathrm{C}$, the percentage of dye fixation was higher with sample $\mathrm{C}$ of the silicone-modified acrylate emulsion adhesive, as shown in Fig. 4. The percentage of dye fixation approached $93.09 \%$; it had increased by $1.83 \%$ of that observed before the adhesive was added into the paste. Importing a benzene ring into the silicone-modified acrylate emulsion adhesive, which includes a hard monomer of St, could increase the combined functions of the polymers and the hydrophobic bond of the polyester or dyes. However, this import resulted in the fabric feeling hard and did not increase the percentage of dye fixation, especially when too much benzene ring (such as E) was imported. As the soft monomer, when the proportion of octamethyl cyclo tetrasiloxane (D4) increased, the percentage of dye fixation also increased. However, importing less D4 resulted in a hard handle of the fabric. The content of St and D4 among the silicone-modified acrylate emulsion adhesive influenced the hand feel of the fabrics and the percentage of dye fixation. When the proportion of soft monomer was larger, they had the advantages of strong viscosity and good handle. The disadvantages included unsatisfactory rubbing fastness, a poor adhesion with the polyester fibres and easy removal of dyes when soaping. On the contrary, the proportion of hard monomer was larger and there was good rubbing fastness, poor adhesion and stiffness of the printed area. Additionally, the fastness of the parcelling dyes declined, and the dyes were easy to remove when soaping [12].

Table 2 shows the effect of the K/S value and the colour features with the different silicone-modified acrylate emulsion adhesive. The temperature of curing was $190^{\circ} \mathrm{C}$.

Table 2 shows that the brightness $\left(L^{*}\right)$ of the soaped samples increased and the values of $a^{*}$ and $K / S$ decreased. The colour brilliance of the samples improved because the dispersed dye molecules on the PET fabric surface or deposit between fabrics 


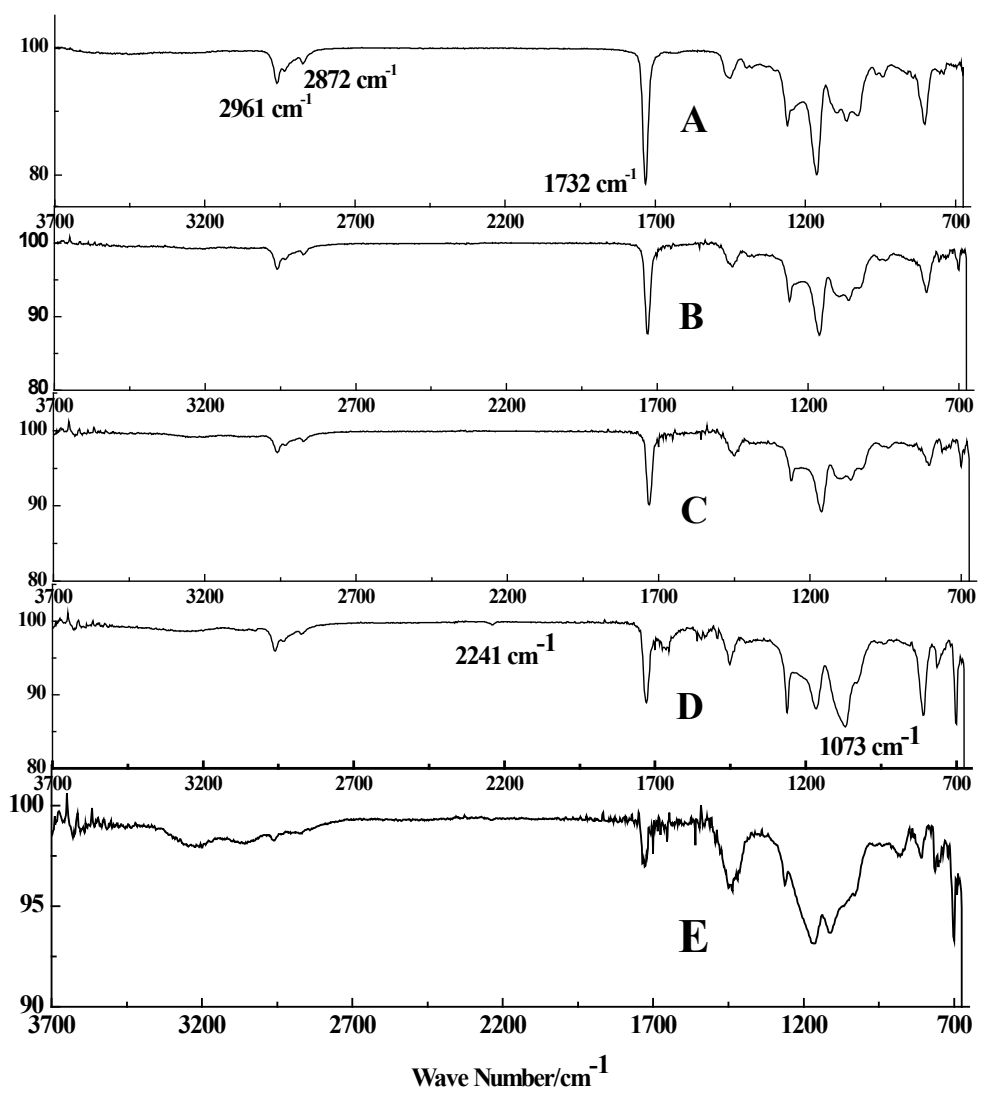

Figure 3. FTIR of the silicone-modified acrylate emulsion membrane

Table 2. Effect of silicone-modified acrylate emulsion adhesive on $K / S$ values and the colour features of printed fabric.

\begin{tabular}{|c|c|c|c|c|c|c|c|c|c|}
\hline \multirow{3}{*}{$\begin{array}{l}\text { Functional } \\
\text { polymers }\end{array}$} & \multicolumn{6}{|c|}{ Colour features } & \multicolumn{3}{|c|}{$K I S$} \\
\hline & \multicolumn{3}{|c|}{ Before soaping } & \multicolumn{3}{|c|}{ After soaping } & \multirow{2}{*}{$\begin{array}{l}\text { Before } \\
\text { soaping }\end{array}$} & \multirow{2}{*}{$\begin{array}{c}\text { After } \\
\text { soaping }\end{array}$} & \multirow{2}{*}{ RF } \\
\hline & $L^{*}$ & $a^{*}$ & $b^{*}$ & $L^{*}$ & $a^{*}$ & $b^{*}$ & & & \\
\hline$A$ & 41.35 & 0.79 & -40.51 & 43.05 & 0.38 & -40.93 & 9.98 & 8.78 & 0.88 \\
\hline$B$ & 38.38 & 2.35 & -40.76 & 40.42 & 1.30 & -40.57 & 12.40 & 10.15 & 0.82 \\
\hline C & 37.93 & 2.77 & -41.43 & 39.76 & 1.79 & -41.23 & 12.44 & 10.79 & 0.87 \\
\hline $\mathrm{D}$ & 38.25 & 2.30 & -41.16 & 40.65 & 1.00 & -41.02 & 12.58 & 10.98 & 0.87 \\
\hline$E$ & 39.44 & 1.33 & -40.68 & 41.86 & 0.11 & -40.11 & 11.48 & 9.40 & 0.82 \\
\hline None & 39.08 & 2.40 & -41.87 & 40.65 & 1.76 & -41.89 & 11.75 & 10.12 & 0.86 \\
\hline
\end{tabular}

were removed during the soaping process. Comparing the $\mathrm{K} / \mathrm{S}$ values of printed fabric containing different silicone-modified acrylate emulsion adhesives, the property of the adhesive could be affected by the value of colour features and $K / S$. When the printing paste contained adhesive $C$, the values of $K / S$ and RF were the highest, regardless of whether there was soaping, which was consistent with the effect of the percentage of dye fixation.

Silicone-modified acrylate emulsion adhesive affected the colour features because they formed a certain thickness of transparent thin film on the surface of the fabric, which resulted in a reflection peak in the region of the yellow and green wavelengths [13-15]. Compared with the absence of siliconemodified acrylate emulsion adhesive, the values of $L^{*}$ and $a^{*}$ were trended downward, while the value of $b^{*}$ increased, which

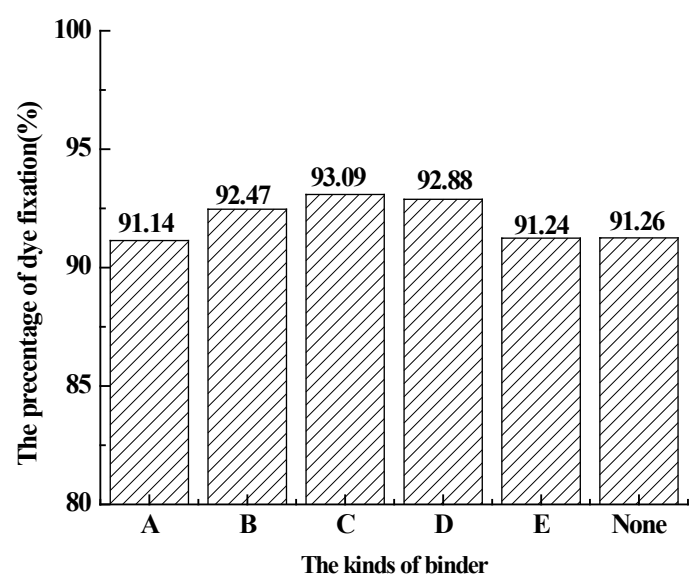

Figure 4. Effect of different adhesives for the percentage of dye fixation 
led to the reduced vividness of colour and lustre, and the tonal hue showed red.

Fig. 5 shows the effect of sublimation of the dye for the curing temperature. Whether or not the silicone-modified acrylate emulsion adhesives were added, there was no effect on the sublimation of the dye on the surface of fabric when the curing temperature was $180^{\circ} \mathrm{C}$. However, the silicone-modified acrylate emulsion adhesive could significantly prevent the dye sublimation when the curing temperature was $190^{\circ} \mathrm{C}$. The amounts of dye sublimation of the sample with and without the silicone-modified acrylate emulsion adhesive were $8.5200 \mathrm{mg}$ and $3.5550 \mathrm{mg}$, respectively. With the curing temperature increased, the silicone-modified acrylate emulsion adhesive had less effect on the reduction of the dye sublimation, although the sublimation became intensified. Disperse dye 79, with good sublimation fastness, had good heat resistance at temperatures less than $180^{\circ} \mathrm{C}$. Meanwhile, the dye tended to sublimate because of increased dye thermal motion at $190^{\circ} \mathrm{C}$. The reason was that the silicone-modified acrylate emulsion adhesive on the surface interacted with the fabric and dye and hindered the sublimation.

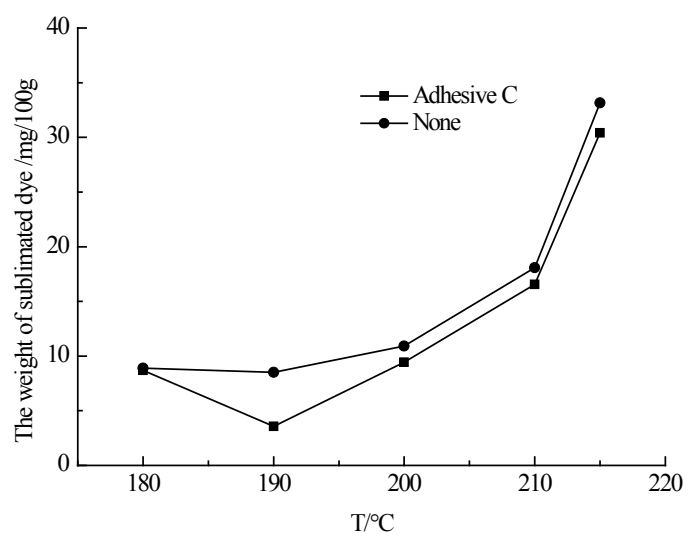

Figure 5. Effect of adhesive on dye sublimation

\subsection{Low-emission printing and direct printing}

The values of $K / S$ and $b^{*}$ of the samples were decreased relative to before the reduction cleaning, which resulted in an increase in the colour brilliance, i.e., becoming red, as shown in Table 3. The colour brilliance sample, with a low value of
$K / S$, obviously improved when the printing process was direct printing. With the low-emission printing process, the RF of the sample was as high as 0.86 , and the sample had the highest $K / S$ value. Meanwhile, when using direct-printing-1 and -2 processes, the RF was $0.71-0.73$. The low-emission printing media that choose the synthetic thickener PTF-A had a high yield of forming thickening agent, and only $1 \%$ of the dosage was thinner, which shortened the diffusion distance of the dye and fabric. However, traditional direct printing choose natural thickener, alginate, and CMC had a low yield of formed thickening agent, with a dosage of $3 \%$ and $4 \%$, respectively. The natural thickener was associated with the strong interactions of dye, which led to more dye residues in the paste than with the synthetic thickener. The synthetic thickener PTF-A did not applied on direct printing, result from the viscosity of thickener had sharp decline with commodity disperse dye. Using synthetic thickener PTF-A as the printing paste could save much water because the use of much less the disperse dye on the PET fabric surface led to a task of reduction cleaning and reduced the wastewater discharge.

The sample with the low-emission printing as the printing paste obtained excellent colour fastness with the staining and colour change at a level 4 or higher and wet and dry rubbing fastness at a level 4 or higher before reduction cleaning, as shown in Table 4; these results were consistent with the higher RF, and the disperse dye on the fabric surface was minimal. The soaping fastness of the sample was at a level 4-5 when the process was direct printing with reduction clearing $\left(85^{\circ} \mathrm{C}, 15 \mathrm{~min}\right)$, $\rightarrow$ Water washing $\rightarrow$ Drying. Therefore, these results reveal that the low-emission printing could avoid the heavy task of water cleaning, which results in low water resource consumption and wastewater discharge.

\subsection{Application and advantages of low-emission printing}

With direct-printing process, the absorbance value of the reduction cleaning residual liquid was larger and the colour was darker than with the low-emission printing, as shown in Fig. 6. For example, the concentration of dye in the wastewater was $50.68 \mathrm{mg} / \mathrm{L}$ when using direct-printing-2 process; with lowemission printing, it was $2.62 \mathrm{mg} / \mathrm{L}$, which was decreased by approximately 19 times than with direct printing. The amount of the residue when using low-emission printing process was smaller than with either direct-printing-1and -2 , which

Table 3. Effect of the $K / S$ value and the colour features of printed fabric with different printing processes.

\begin{tabular}{|c|c|c|c|c|c|c|c|c|c|}
\hline \multirow{3}{*}{ Printing ways } & \multicolumn{6}{|c|}{ Colour features } & \multicolumn{3}{|c|}{$K I S$} \\
\hline & \multicolumn{3}{|c|}{ Before soaping } & \multicolumn{3}{|c|}{ After soaping } & \multirow{2}{*}{$\begin{array}{l}\text { Before } \\
\text { soaping }\end{array}$} & \multirow{2}{*}{$\begin{array}{c}\text { After } \\
\text { soaping }\end{array}$} & \multirow{2}{*}{ RF } \\
\hline & $L^{*}$ & $a^{*}$ & $b^{*}$ & $L^{*}$ & $a^{*}$ & $b^{*}$ & & & \\
\hline $\begin{array}{l}\text { Low-emission } \\
\text { printing }\end{array}$ & 39.08 & 2.40 & -41.87 & 40.65 & 1.76 & -41.89 & 11.75 & 10.12 & 0.86 \\
\hline Direct printing-1 & 42.16 & -1.02 & -30.52 & 51.51 & -2.52 & -37.87 & 6.64 & 4.82 & 0.73 \\
\hline Direct printing-2 & 42.36 & -0.95 & -30.70 & 52.10 & -2.82 & -38.18 & 6.73 & 4.76 & 0.71 \\
\hline
\end{tabular}


Table 4. Effect of thickener on colour fastness of printed PET.

\begin{tabular}{|c|c|c|c|c|c|c|}
\hline \multirow{2}{*}{ Printing } & \multicolumn{2}{|c|}{ Wash fastness } & \multicolumn{4}{c|}{ Rub fastness } \\
\cline { 2 - 7 } & \multicolumn{2}{|c|}{} & \multicolumn{2}{c|}{ Before soaping } & \multicolumn{2}{c|}{ After soaping } \\
\cline { 2 - 7 } & Staining & Change & Dry & Wet & 5 & $4-5$ \\
\hline Low-emission printing & 4 & $4-5$ & $4-5$ & 4 & $4-5$ & $4-5$ \\
\hline Direct-printing-1 & $4-5$ & $4-5$ & $4-5$ & $4-5$ & $4-5$ & $4-5$ \\
\hline Direct-printing-2 & $4-5$ & $4-5$ & $4-5$ & $4-5$ & 5 \\
\hline
\end{tabular}

Table 5. Effect of adhesive on the wastewater of printed PET.

\begin{tabular}{|c|c|c|c|}
\hline \multirow{2}{*}{ Printing way } & \multirow{2}{*}{ Low-emission printing } & $\mathbf{1}$ & $\mathbf{2}$ \\
\cline { 3 - 4 } & & 9.0 & 9.0 \\
\hline $\mathrm{pH}$ & 6.7 & 919 & 966 \\
\hline COD (mg/L) & 976 & 16.64 & 21.62 \\
\hline
\end{tabular}

decreased $70.0 \%$ and $76.9 \%$, respectively, as shown in Table 5.

In conclusion, the low-emission printing can be a green printing processing method. This method has advantages of a higher $K / S$ value, an increased utilization rate of dye, an improved amount of disperse dye on the fabric surface, a reduced burden of reductive cleaning, and a decreased production of wastewater and residue emissions. All of these advantages have the potential to solve the technology bottleneck of high water consumption and production of wastewater and residue discharge.

The silicone-modified acrylate emulsion adhesive adhered to the surface of the fabric, presenting a thin film structure on the surface of the fabric, as shown in Fig. 7, which is similar to the function of a binder in pigment printing. Although a part

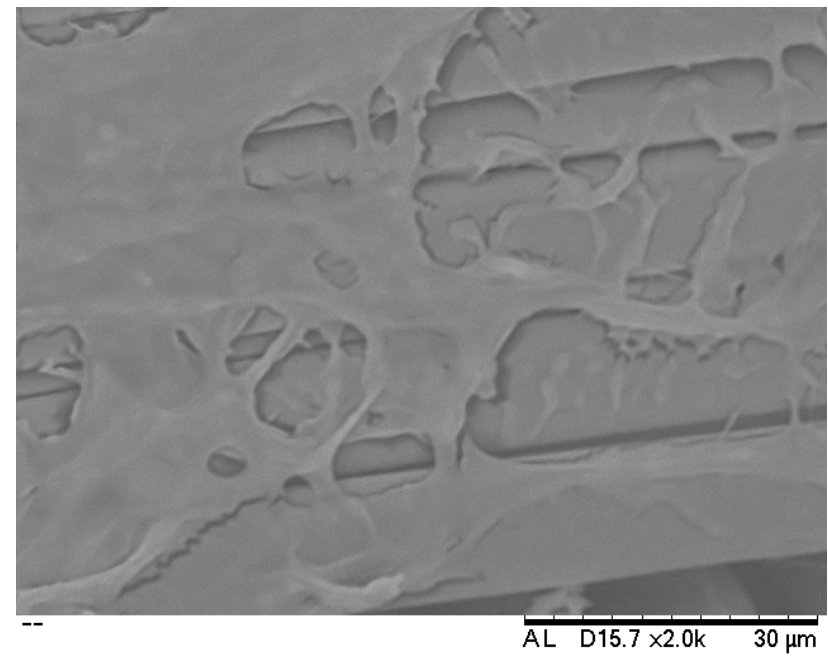

(a) Before soaping

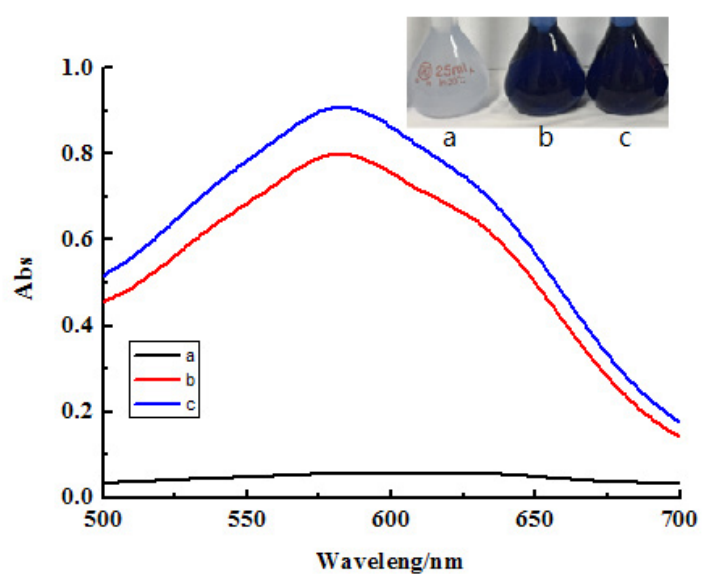

Figure 6. Effect of absorbance on reduction cleaning liquid (a: lowemission printing; b: direct-printing-1; c: direct-printing-2)

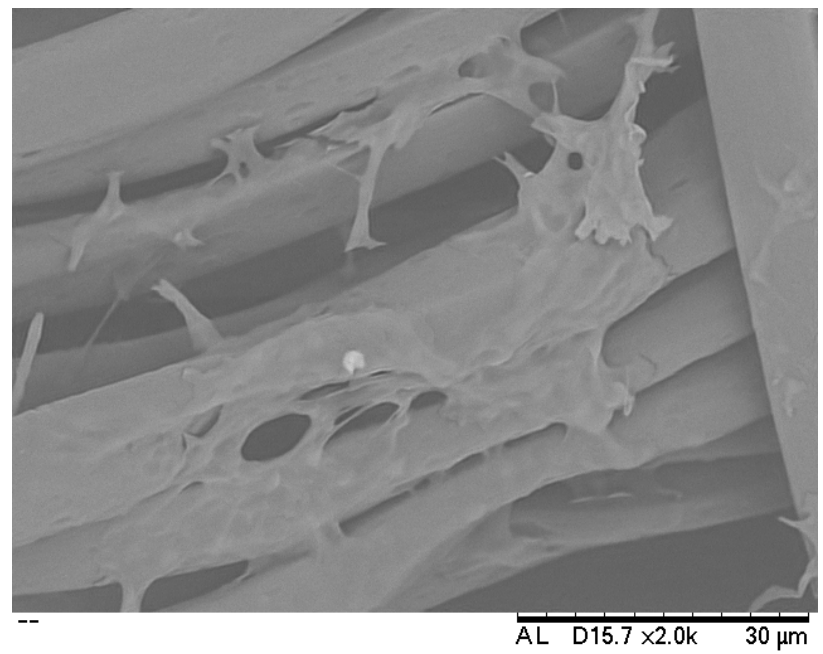

(b) After soaping

Figure 7. Surface topography of the adhesive film on the surface of PET. 
of the film of the silicone-modified acrylate emulsion adhesive fell off after the sample reductive clearing, it had no effect on the colour. Moreover, the dye concentration of the wastewater was low because less disperse dye existed on the surface of the fibre. The fabric that was printed by low-emission printing showed soft tactility because the solid content in the paste was extremely low beside the disperse dye, which did little affect the handle of the fabric.

The low-emission printing, a type of green and low-pollution printing process, can primarily solve three technological problems. First, a liquid disperse dye with a low content of surfactant, which has less effect on reducing the viscosity of the thickener, is the foundation of this low-emission mechanism. The anti-electrolytic property of the anionic synthetic thickener was poor, because the electrolytic could lead to a sharp decline in viscosity, such as dyes, surfactants, acids and salts. Additionally, commodity disperse dye contained a large number of anionic disperse agents (MF), which led to increasing the dosage of the synthetic thickener to meet the requirements of the rheological property of the paste. The contradiction between the synthetic thickener and electrolyte was needed to solve the primary problem of low-emission mechanism. Second, preparations of the silicone-modified acrylate emulsion was another key point; the silicone-modified acrylate emulsion increased the percentage of dye fixation of the disperse dye and reduced the retention rate. The properties that included the proportion of monomers and the interaction with the dye and fabric of the silicone-modified acrylate emulsion adhesive directly affected the percentage of dye fixation and handle. Third, by synthesized silicone-modified acrylate emulsion and used adhesive, the soft handle and good colour fastness were also satisfactory. Low-emission printing, which improved the utilization rate of dye and reduced the dye concentration of the wastewater and residue emissions, solved the problem of the heavy burden of reductive clearing because the paste contained silicone-modified acrylate emulsion adhesive and less printed media.

During the curing process at high temperatures, the siliconemodified acrylate emulsion gathered, demulsified and completed adhesion, deformation and film formation on the surface of the fabric; also, the disperse dye finished colouration [16]. The silicone-modified acrylate emulsion improved the percentage of dye fixation and effectively prevented the dye from escaping into the air because of the binding of the dye under the condition of curing (such as at $190^{\circ} \mathrm{C}$ ).

\section{Conclusion}

In this study, we have proposed low-emission printing, which combines the advantages of both direct printing and pigment printing. The low-emission printing paste contained liquid disperse dye, thickener and adhesive. We synthesized silicone-modified acrylate emulsion and selected by adjusting the proportion of the soft and hard monomers for adhesive coating of PET fibres. The silicone-modified acrylate emulsion adhesive coating agent had many excellent characteristics, such as softness, elasticity, good adhesion, and wear resistance when it was used as the binder for PET fibres with the lowemission printing process. The properties of the siliconemodified acrylate emulsion affected the value of $K / S$ and the colour features. The silicone-modified acrylate emulsion (such as sample C) can effectively improve the percentage of dye fixation on PET fibres when applied to printing. Moreover, the silicone-modified acrylate binder could significantly prevent dye sublimation. With the low-emission printing process, printed PET fibres could achieve good fastness to the wash, staining and colour change at a level 4 and above, and wet and dry rubbing fastness at a level 4 and above. The dye concentration of the wastewater was very low, only $2.62 \mathrm{mg} / \mathrm{L}$, which was decreased by 19 times that when using the direct-printing process. Low-emission printing combines the advantages of direct and pigment printing. The silicone-modified acrylate emulsion adhesive can effectively increase the percentage of dye fixation and decrease the dye concentration of wastewater and the amount of waste residue.

\section{Acknowledgements}

This work was supported by the Jiangsu Province Education Department (Nos. BE2014058) and Changzhou Vocational Institute of Textile and Garment Research Project (CFK201502) and Jiangsu Advanced Textile Engineering Technology Center Research Project (XJFZ/2016/17).

\section{References}

[1] Schwindt, W, Faulhaber, G, Moore, A. J. (2010). Resinbonded pigment printing and dyeing. Coloration Technology, 2(1), 33-41.

[2] Warburton, C. E. Jr. (1975). Crockfastness of polyacrylate textile pigment-printing binders: effect of binder mechanical properties and adhesion to fabric. Journal of Adhesion, 7(2), 109-119.

[3] Jiang, X., Hu, H., Bai, Y., et al. (2013). Synthesis and properties of the vinyl silicone oil modified polyacrylate core-shell latex as a binder for pigment printing. Journal of Adhesion Science \& Technology, 27(2), 154-164.

[4] Hamilton, L. E, Chiweshe, A. (1998). Textile pigment printing binders prepared by modifying wheat gluten with methyl acrylate. Starch-Stärke, 50(5), 213-218.

[5] lqbal, M., Mughal, J., Sohail, M., Moiz, A., Ahmed, K., et al. (2012). Comparison between pigment printing systems with acrylate and butadiene based binders. Journal of Analytical Sciences Methods \& Instrumentation, 2(2), 87-91.

[6] Sui, Z., Pang, W., Song, J., Zhang, L. (2014). Preparation of silicone-modified acrylic ester emulsion adhesive with core-shell structure. Asian Journal of Chemistry, 26(14), 4435-4438.

[7] Jiang, X., Gu, J., Tian, X., Yang, Y. (2011). Synthesis of the hydroxyl-containing poly(dimethyl siloxane) modified polyacrylate core-shell latex and the application as a novel binder for pigment printing of fabric. Journal of Dispersion Science \& Technology, 32(9), 1266-1272.

[8] Cao, S., Liu, B., Deng, X., Li, S. (2005). Core/shell particles containing 3-(methacryloxypropyl)-trimethoxysilane in the shell: synthesis, characterization, and application. Macromolecular Bioscience, 5(7), 669-76. 
[9] Yen, M. S, Tsai, H. C, Hong, P. D. (2010). Effect of soft segment composition on the physical properties of nonionic aqueous polyurethane containing side chain PEGME. Journal of Applied Polymer Science, 105(3), 1391-1399.

[10] Yen, M. S., Tsai, H. C., Hong, P. D. (2006). The physical properties of aqueous cationic-nonionic polyurethane with poly (ethylene glycol methyl ether) side chain and its blend with aqueous cationic polyurethane. Journal of Applied Polymer Science, 100(4), 2963-2974.

[11] Li, M., Gao, A., Wu, L., Fu, D., Kongliang X. (2016). Crosslinking formulations based on novel reactive disperse dyes for printing cotton fabrics. Textile Research Journal, 87(17), 2127-2132.

[12] Chern, C. S., Lin, S. Y., Chen, L. J., Wu, S. C. (1997). Emulsion polymerization of styrene stabilized by mixed anionic and nonionic surfactants. Polymer, 38(8), 19771984.
[13] Meng, Y., Tang, B., Xiu, J., Zheng X., Ma W. (2015). Simple fabrication of colloidal crystal structural color films with good mechanical stability and high hydrophobicity. Dyes \& Pigments, 123, 420-426.

[14] Xi, L. I, Liu, Y., Jiawen, L. I., Wen-hao H. (2010). Influence of the optical multi-film thickness on the saturation of the structural color displayed. Advances in Natural Science, 2(3), 317-323.

[15] Cathell, M. D., Schauer, C. L. (2007). Structurally colored thin films of Ca2+-cross-linked alginate. Biomacromolecules, 8(1), 33-41.

[16] Giesen, V., Eisenlohr, R. (1994). Pigment printing. Coloration Technology, 1994, 24(1), 26-30. 